University of Nebraska - Lincoln

DigitalCommons@University of Nebraska - Lincoln

\title{
Analysis of Human Skin Emanations by Gas Chromatography/ Mass Spectrometry. 1. Thermal Desorption of Attractants for the Yellow Fever Mosquito (Aedes aegypti) from Handled Glass Beads
}

\author{
Ulrich R. Bernier \\ University of Florida, ubernier@gainesville.usda.ufl.edu \\ Matthew M. Booth \\ University of Florida \\ Richard A. Yost \\ University of Florida
}

Follow this and additional works at: https://digitalcommons.unl.edu/usdaarsfacpub

Part of the Agricultural Science Commons

\begin{abstract}
Bernier, Ulrich R.; Booth, Matthew M.; and Yost, Richard A., "Analysis of Human Skin Emanations by Gas Chromatography/Mass Spectrometry. 1. Thermal Desorption of Attractants for the Yellow Fever Mosquito (Aedes aegypti) from Handled Glass Beads" (1999). Publications from USDA-ARS / UNL Faculty. 945. https://digitalcommons.unl.edu/usdaarsfacpub/945
\end{abstract}

This Article is brought to you for free and open access by the U.S. Department of Agriculture: Agricultural Research Service, Lincoln, Nebraska at DigitalCommons@University of Nebraska - Lincoln. It has been accepted for inclusion in Publications from USDA-ARS / UNL Faculty by an authorized administrator of DigitalCommons@University of Nebraska - Lincoln. 


\title{
Analysis of Human Skin Emanations by Gas Chromatography/Mass Spectrometry. 1. Thermal Desorption of Attractants for the Yellow Fever Mosquito (Aedes aegypti) from Handled Glass Beads
}

\author{
Ulrich R. Bernier, ${ }^{,+t, \neq}$ Matthew M. Booth, ${ }^{\dagger}$ and Richard A. Yost ${ }^{\dagger}$ \\ Department of Chemistry, University of Florida, P.O. Box 117200, Chemistry Laboratory Building, Gainesville, Florida 32611, \\ and United States Department of Agriculture-Agricultural Research Service, Center for Medical, Agricultural, and Veterinary \\ Entomology, 1600 SW 23rd Drive, Gainesville, Florida 32608
}

Handled glass has the ability to collect and concentrate nonaqueous human skin emanations while minimizing the collection of aqueous perspiration. Compounds originating from the skin and collected on glass have previously been found to attract the Aedes aegypti species of mosquito. Therefore, glass beads were used as the medium to collect skin emanations from humans for subsequent chemical analysis. This process consisted of a 5-15-min collection of sample on glass beads, followed by loading the beads into a gas chromatograph (GC) injector insert for subsequent desorption of the collected compounds onto the GC column. After cryofocusing by liquid nitrogen at the head of the column, the thermally desorbed compounds were analyzed by GC/ MS. Microscale purge and trap introduction was also used to provide complementary information. In this case, the beads are held in a round-bottom flask, purged with nitrogen, and heated as the concentrator collects the headspace above the beads. The chromatograms produced by both of these sample introduction methods demonstrate good resolution of a complex sample. Cryofocusing volatiles from handled glass allowed identification of lactic acid, aliphatic fatty acids, and other polar to nonpolar compounds of moderate volatility while purge and trap allowed detection of nonpolar to moderately polar compounds of high volatility. Published on Web 11/25/1998
M osquitoes are attracted to humans by various stimuli. ${ }^{1} \mathrm{H}$ eat and vision are two important stimuli utilized by mosquitoes to locate a host, 2,3 but olfaction is probably the most important stimulus. This stimulus involves host location by means of detecting volatile chemicals emanated from the host and following these chemical odor plumes upstream to the source. Two compounds that have been found to elicit attraction in Aedes aegypti (L.) are L-lactic acid and carbon dioxide. ${ }^{2,4-8}$ In addition to these, other compounds emanating from the skin, which are still undiscovered, also play a role in the attraction of this species of mosquito. ${ }^{9}$

* Corresponding author: (Phone) 352-374-5917; (Fax) 352-374-5922; (e-mail) ubernier@gainesville.usda.ufl.edu.

+ University of Florida.

₹ Current affiliation is USDA-ARS. Some of this work performed while at the University of Florida.

(1) Bar-Zeev, M .; M aibach, H. I.; Khan, A. A. J. M ed. Entomol. 1977, 14, 11320.

(2) Price, G. D.; Smith, N.; Carlson, D. A. J. Chem. Ecol. 1979, 5, 383-95.

(3) Bidlingmayer, W. L. J. Am. M osq. Control Assoc. 1994, 10, 272-9.

(4) Acree, F.; Turner, R. B.; Gouck, H. K.; Beroza, M.; Smith, N. Science 1968, $161,1346-7$.

(5) Smith, C. N.; Smith, N .; Gouck, H. K.; Weidhaas, D. E.; Gilbert, I. H.; M ayer, M. S.; Smittle, B. J.; Hofbauer, A. Ann. Entomol. Soc. Am. 1970, 63, 76070

(6) Carlson, D. A.; Smith, N.; Gouck, H. K.; Godwin, D. R. J. Econ. Entomol. 1973, 66, 329-31.

(7) Gillies, M. T. Bull. Entomol. Res. 1980, 70, 525-32.

(8) Takken, W. Insect Sci. I ts Appl. 1991, 12, 287-95.

(9) Schreck, C. E.; Smith, N.; Carlson, D. A.; Price, G. D.; Haile, D.; Godwin, D. R. J. Chem. Ecol. 1981, 8, 429-38.

Analytical Chemistry, Vol. 71, No. 1, January 1, 19991 
The problem that plagues the chemical analysis and sampling of human emanations is the large amount of water, in the form of perspiration, that is given off by the human body. Loading of water onto a fused-silica open tubular column is detrimental to the column stationary phase, leading to broad peaks, column degradation, and shifts in compound retention times.

The goal of this study is to develop a sampling method that maintains the integrity of the sample and allows detection of volatile candidate attractants. Little or no chemical modification of the skin emanations is desired such that detection of volatiles is as comparable as possible to that of the detection by mosquito sensilla. While in-flight and seeking a host, the mosquito chemosensilla are not responding to dissolved compounds; they are instead responding to airborne components which specifically cause the activation or host-seeking behavior. Therefore, in the initial phase of this work, solvent extraction was avoided, as was the use of sorbent or solid-phase traps. It should also be noted that the purpose is not to identify the constituents in perspiration, ${ }^{10}$ but rather volatile compounds that are candidates as attractants of Ae. a egypti; these volatiles may not be present in the perspiration aqueous phase. Previous analyses of volatiles from perspiration have demonstrated the complexity of human odor and perspiration. ${ }^{11-13}$

M osquito attractants, including lactic acid, can be transferred to glass via handling a glass object. ${ }^{9}$ The quantitative and qualitative assessment of attraction is made by counting mosquitoes attracted to this object or sample, over a specific time, in an olfactometer. ${ }^{14,15}$ Glass Petri dishes handled by humans are attractive in olfactometer experiments and retain their attraction for up to 6 h. ${ }^{16}$ Furthermore, differences in attraction between human subjects are reflected in the attraction of the Petri dishes handled by these subjects. Therefore, chemical analysis of human skin volatiles by GC/MS may be able to define the observed differences between human subjects.

Storage of samples and duration of the attraction once the sample is deposited on glass are also concerns. It is known that volatile skin emanations containing compounds that are attractive to mosquitoes are amenable to cold-trapping. Experiments involving cold-trapping of emanations in an air stream provided, after reconstitution of the sample, $\sim 60 \%$ attraction compared to direct introduction of emanations into an olfactometer. ${ }^{2}$ Ethanol-extracted skin samples were successfully used to attract Ae. aegypti; ${ }^{17}$

(10) Yokoyama, Y.; Aragaki, M .; Sato, H.; Tsuchiya, M. Anal. Chim. Acta 1991, 246, 405-11.

(11) Ellin, R. I.; Farrand, R. L.; Oberst, F. W.; Crouse, C. L.; Billups, N. B.; Koon, W. S.; M usselman, N. P.; Sidell, F. R. J. Chromatogr. 1974, 100, 137-52.

(12) Sastry, S. D.; Buck, K. T.; Janák, J.; Dressler, M.; Preti, G. In Biochemical Applications of M ass Spectrometry, First Supplementary Volume; Waller, G. R., Dermer, O. C., Eds.; John Wiley \& Sons: Chichester, England, 1980; pp 1086-129.

(13) Sommerville, B. A.; M cCormick, J. P.; Broom, D. M. Pestic. Sci. 1994, 41, 365-8.

(14) Schreck, C. E.; Gouck, H. K.; Smith, N. J. Econ. Entomol. 1967, 60, 118890.

(15) Posey, K. H.; Barnard, D. R.; Schreck, C. E. J. Med. Entomol. 1998, 35 , $330-4$.

(16) Schreck, C. E.; Kline, D. L.; Carlson, D. A. J. Am. M osq. Control Assoc. 1990, $6,406-10$.

(17) Geier, M .; Sass, H.; Boeckh, J. In M osquito-H ost Interactions-Ciba F oundation Symposium 200; Bock, G. R.; Cardew, G., Eds.; John Wiley \& Sons: Chichester, England, 1996, pp 132-48. however, the active components have not yet been reported. We describe a different approach to the collection of volatile skin emanations which has led to the identification of additional compounds that are potential mosquito attractants.

\section{EXPERIMENTAL SECTION}

Injection Port Modification. A Varian 3400 GC fritted glass injection liner (Varian, Walnut Creek, CA) was installed in reversed orientation into the GC injection port. Up to 12 beads could be placed between the frit and the injection port septum. The frit kept the beads from dropping down onto the column entrance and provided a means for volatile emanations to be loaded onto the column. The injector end of the column extended into the injector insert just below the frit. The injection port was operated in splitless mode for the duration of the experiment.

Sample Collection and Cryofocusing. Some experiments consisted of a $1.0-\mu \mathrm{L}$ injection of a $120 \mathrm{ng} / \mu \mathrm{L}$ L-lactic acid standard (Aldrich Chemical Co., M ilwaukee, WI) in methanol (Aldrich Chemical Co.). All other experiments employed glass beads of $2.9 \mathrm{~mm}$ ( $0.115 \mathrm{in}$.) in diameter that were rubbed in the palms of the hands for 10-15 min by a human subject prior to being loaded into a Varian 3400 injector insert. It has been documented that the length of handling time beyond $10 \mathrm{~min}$ does not affect appreciably the quantity of deposited emanations. ${ }^{9}$ The beads were then transferred into the insert either by the subject directly or with the use of clean forceps. The insert was handled with gloves to avoid contamination from direct contact with human skin. Experiments were performed using from 5 to 8 beads, although a maximum of 12 beads can be used. After the beads were loaded into the insert, the insert was placed into the injection port held at $25^{\circ} \mathrm{C}$ to minimize sample loss from evaporation.

Repetitive analyses required sufficient cooling time to allow the injection port and insert to cool to a low enough temperature for safe handling with nylon and disposable gloves. Dust-Off (difluoroethane; SIS, Ringoes, NJ) was used to speed the cooling of the injection insert to facilitate more rapid sample throughput. The cap, septum, and needle guide were replaced to seal the injection port with the insert back into position. The helium head pressure was set to 0 psig during the changing and placement of samples in the injection port.

A Styrofoam cup (12 oz) filled with liquid nitrogen was placed in the oven such that $\sim 8 \mathrm{~cm}$ of column could be looped in the container $\sim 15 \mathrm{~cm}$ below the point where the column passes from injection port to oven. The helium pressure was then increased to 20 psig and the initial desorption phase started. The injection port was ramped from 25 to $250^{\circ} \mathrm{C}$ over $7.5 \mathrm{~min}$ and then held at $250{ }^{\circ} \mathrm{C}$ for $2.5 \mathrm{~min}$. Throughout the cryofocusing phase, the $\mathrm{GC}$ oven was set at $25^{\circ} \mathrm{C}$ and the transfer line set at $40{ }^{\circ} \mathrm{C}$.

Chromatographic Parameters. Upon completion of the cryofocusing program, the oven was heated slightly to either 30 or $40{ }^{\circ} \mathrm{C}$ by the analysis program. Once an equilibrium oven temperature was reached, the cup was removed and the analysis was started. For the Carbowax column described below, the GC analysis ramp consisted of a 1.0 -min hold at $30^{\circ} \mathrm{C}$, a 12.0 -min ramp at $15^{\circ} \mathrm{C} / \mathrm{min}$ up to $210{ }^{\circ} \mathrm{C}$, and then a hold at $210{ }^{\circ} \mathrm{C}$ for 17.0 $\min$. The transfer line was concurrently ramped, after a 1.0-min hold at $40^{\circ} \mathrm{C}$, up to $220^{\circ} \mathrm{C}$ at $15^{\circ} \mathrm{C} / \mathrm{min}$, and a 17.0-min hold at

2 Analytical Chemistry, Vol. 71, No. 1, January 1, 1999 
$220{ }^{\circ} \mathrm{C}$. The total analysis time was $30 \mathrm{~min}$. Experiments were performed initially with a $20 \mathrm{~m} \times 0.25 \mathrm{~mm}$ i.d. Carbowax column $\left(d_{\mathrm{f}}=0.25 \mu \mathrm{m}\right)(J \& W$ Scientific, Folsom, CA). Later experiments employed either a HP5 (Hewlett-Packard, Atlanta, GA) column or HP-FFAP (free fatty acid polar) column. Both columns were $25 \mathrm{~m} \times 0.20 \mathrm{~mm}$ i.d., with $d_{f}=0.33 \mu \mathrm{m}$. The oven temperature ramp for the HP5 column consisted of a 1.0 -min hold at $40{ }^{\circ} \mathrm{C}$, followed by a 10.6 -min ramp at $17^{\circ} \mathrm{C} / \mathrm{min}$, with a hold at $220^{\circ} \mathrm{C}$ for $23.4 \mathrm{~min}$ ( $35 \mathrm{~min}$ total run time). When the FFAP column was used for single-stage mass spectrometry experiments, the $\mathrm{GC}$ run time was $45.0 \mathrm{~min}$, consisting of a $1.0-\mathrm{min}$ hold at $40^{\circ} \mathrm{C}$, followed by a 17.7 -min ramp at $11^{\circ} \mathrm{C} / \mathrm{min}$ to $235^{\circ} \mathrm{C}$, with a 26.3 -min hold at that temperature. Experiments involving $\mathrm{MS} / \mathrm{M} \mathrm{S}$ consisted of a 40.0 -min program with a 1.0 -min hold at $40^{\circ} \mathrm{C}$, a 32.0 -min ramp at $5{ }^{\circ} \mathrm{C} / \mathrm{min}$ to $200{ }^{\circ} \mathrm{C}$, and a 7.0 -min hold at $200{ }^{\circ} \mathrm{C}$. The head pressure of helium carrier gas was adjusted for each column to produce a column linear velocity equal to or just greater than twice the theoretical optimum average linear velocity for minimum plate height under isothermal conditions. The final temperature was used in calculations performed by a BASIC program based on theoretical equations. ${ }^{18,19}$

Mass Spectrometric Ionization Modes and Parameters. The TSQ 70 triple-quadrupole mass spectrometer (Finnigan-M AT, San Jose, CA) was operated in positive ion chemical ionization $(\mathrm{PCl})$, negative ion chemical ionization $(\mathrm{NCl})$, pulsed positive ion negative ion chemical ionization (PPINICI), or electron ionization (EI) mode. Use of PPINICI allowed concurrent detection of both positive and negative ions by alternating between positive and negative ion modes on successive scans. ${ }^{20}$ When the file containing the PPINICI analysis is deconvoluted, two separate reconstructed ion chromatograms (RICS) are produced, one in $\mathrm{PCI}$ mode, the other in $\mathrm{NCl}$ mode. The benefit is that these two modes were acquired at approximately the same time for a particular sample and set of experiment parameters. Both RICs should match well, providing an additional means of identifying the relative molecular mass of unknown compound peaks observed in the chromatograms.

For all $\mathrm{Cl}$ experiments, $(\mathrm{PCl}, \mathrm{NCl}, \mathrm{PPINICl})$, the reagent gas was methane at 1650-1660 mTorr (indicated) pressure. The ion source and manifold temperatures were 150 and $70{ }^{\circ} \mathrm{C}$, respectively, for $\mathrm{Cl}$ and 170 and $70^{\circ} \mathrm{C}$, respectively, for $\mathrm{EI}$. The electron energy for $\mathrm{Cl}$ mode was $100 \mathrm{eV}$ and was $70 \mathrm{eV}$ for $\mathrm{EI}$ mode. For experiments with a single stage of mass spectrometry, the third quadrupole (Q3) was scanned with a scan time of $0.5 \mathrm{~s}$ for $\mathrm{EI}$ and $1.0 \mathrm{~s}$ for PPINICI experiments. A filament emission current of $200 \mu \mathrm{A}$ was used for all analyses. The conversion dynode was set at $-5 \mathrm{kV}$ for positive ion $\mathrm{Cl}$ and $\mathrm{El}$ and $+5 \mathrm{kV}$ for negative ion $\mathrm{Cl}$. The electron multiplier was set at -1000 to $-1200 \mathrm{~V}$. The mass range acquired was $\mathrm{m} / \mathrm{z}$ 35-650 for EI, $\mathrm{m} / \mathrm{z}$ 50-650 for $\mathrm{PCl}$, and $\mathrm{m} / \mathrm{z} 10-650$ for $\mathrm{NCl}$. Prior to analysis the instrument was tuned and calibrated with perfluorotributylamine (PFTBA), and blanks (appropriate number of beads without sample) were analyzed.

(18) Hail, M. E.; Yost, R. A. Anal. Chem. 1989, 61, 2402-10.

(19) Bernier, U. R.; Yost, R. A. J. Chromatogr. Sci. 1993, 31, 358-62.

(20) Hunt, D. F.; Stafford, G. C.; Crow, F. W.; Russell, J. W. Anal. Chem. 1976, 48, 2098-105.
Product mass spectra were produced by selecting a single $\mathrm{m} / \mathrm{z}$ with the first quadrupole (Q1), performing collision-induced dissociation (CID) in the second quadrupole collision cell (Q2), and scanning the third quadrupole (Q3) for resultant fragment ions. In the negative ion experiments, where the precursor ion is formed by $\mathrm{NCl}$, the collision gas was nitrogen at $1.97 \mathrm{mTorr}$ indicated pressure, with a collision energy of $9 \mathrm{eV}$.

Neutral loss mass spectra were generated from positive precursor ions, formed by $\mathrm{PCl}$. The $\mathrm{GC}$ and $\mathrm{MS}$ operating conditions were as described above for the HP-FFAP column used in these analyses. The collision gas for these experiments was argon at 1.2-1.5 mTorr indicated pressure with the collision energy set at $15-19 \mathrm{eV}$.

Microscale Purge and Trap GC Instrumentation and Methods. Some analyses were performed using a microscale purge and trap system. Experiments involved handling 100 glass beads for $10 \mathrm{~min}$. The beads were then transferred to a $100-\mathrm{mL}$ round-bottom flask. The round-bottom flask was attached to the purge and trap system via a $1 / 2^{-i n}$. Cajon fitting (Jax Valve, Jacksonville, FL) to $1 / 4$-in. Swagelok (J ax Valve), further reduced to a $1 / 8$-in. Swagelok fitting. The flask was attached to a port on an ELA2010 canister manifold (Entech Laboratory Automation, Simi Valley, CA).

The canister manifold allowed for $70-100 \mathrm{~mL}$ of volatiles and residual air to be sampled by the ELA2000 concentrator. The concentrator consisted of three stages. The first stage employed a dryer with a gradient of large to small glass beads through the dryer. This served to remove most of the water in the sample. During concentration, this stage is set to $-160{ }^{\circ} \mathrm{C}$ for $3 \mathrm{~min}$ and heats to $-16^{\circ} \mathrm{C}$ as sample is transferred to the second stage. The second stage in these experiments was a Tenax trap. During concentration, the trap was set to $-20^{\circ} \mathrm{C}$ and then heated to 156 ${ }^{\circ} \mathrm{C}$ for desorption of trapped volatiles onto the focusing trap. The cryofocusing trap was set at $-160^{\circ} \mathrm{C}$ for concentration and was heated ballistically (in $10 \mathrm{~s}$ ) to $150{ }^{\circ} \mathrm{C}$ to purge volatiles onto the head of the $\mathrm{GC}$ column.

The GC employed was an HP5890 series I (Hewlett-Packard) with a $30 \mathrm{~m} \times 0.25 \mathrm{~mm}$ i.d. DB-1 column $\left(\mathrm{d}_{\mathrm{f}}=1.0 \mu \mathrm{m}\right)(\mathrm{J} \& \mathrm{~W}$ Scientific). The column oven was initially cryocooled to $35^{\circ} \mathrm{C}$ and held for $3.0 \mathrm{~min}$. Subsequently, the column oven was ramped at $12{ }^{\circ} \mathrm{C} /$ min up to $180^{\circ} \mathrm{C}$, then ramped at $25^{\circ} \mathrm{C} /$ min from 180 to $225^{\circ} \mathrm{C}$, and held at $225^{\circ} \mathrm{C}$ for the remainder of the analysis.

The mass spectrometer used for microscale purge and trap was an Incos 50 (Finnigan-M AT) single-stage quadrupole. The scan rate employed was $0.75 \mathrm{~s} / \mathrm{scan}$. The filament emission current was set at $750 \mu \mathrm{A}$ with an electron energy of $70 \mathrm{eV}$. The electron multiplier was set to $-1200 \mathrm{~V}$. The ion source temperature was set at $180^{\circ} \mathrm{C}$; there is no heater for the manifold. Prior to analysis, the instrument was tuned with PFTBA.

\section{RESULTS AND DISCUSSION}

Initial method development for this approach consisted of using a single 2.9-mm-diameter glass bead fitted to a glass stem. The stem was designed to fit into the crucible inlet of the TSQ 70 directinlet probe. The heatable direct-inlet probe allowed for samples to be placed through a vacuum lock, directly into the mass spectrometer ion source. The bead and stem provided rapid 
analysis of volatile components. Unfortunately, a single bead did not provide adequate sample to detect minor components, nor did the use of a direct-inlet probe and thermal desorption provide sufficient temporal resolution for complex mixture analysis. However, one compound that was readily detectable from a single handled bead/stem was cholesterol, primarily due to the significant $\mathrm{m} / \mathrm{z} 386$ molecular ion and characteristic fragmentation produced in EI analysis. To overcome the problem of insufficient sample size, multiple beads were collected in glass containers for subsequent thermal desorption in the GC oven, with the volatiles purged directly into the ion source via a fused-silica open tubular column. From 2 to 200 beads were tested in various container designs before the decision to use the GC injection port to desorb volatiles from small glass beads followed by GC separation.

Volatiles desorbed from the glass beads have wide desorption profiles; thus, some method of reducing bandwidth before chromatographic separation is needed to significantly improve the chromatographic resolution. This was accomplished either by cryofocusing or by microscale purge and trap. Cryofocusing without purge and trap involved immersing a portion of the column, just after the column exits the injection port, into liquid nitrogen to collect volatiles and focus them into a narrow band. ${ }^{21}$ After focusing, the column was removed from the liquid nitrogen and GC separation conducted. Purge and trap is slightly more complex in that it typically employs one or more additional traps. ${ }^{21}$

Thermal Desorption from Multiple Beads and Cryofocused GC Separation. Cryofocusing GC/MS has been used previously in the detection of volatile emanations from humans with collection and desorption of human emanations on charcoal and refocusing on three successively colder traps. ${ }^{11}$ In contrast, adequate sampling was achieved in the studies reported here by employing liquid nitrogen in a single stage of cold-trapping. The use of on-column cryofocusing solved the problem of wide desorption profiles due to inefficient compound removal from the headspace above the beads in earlier experiments.

When 12 beads were used, the major components of the desorbed sample, i.e., the carboxylic acids, were observed as nonsymmetrical fronted peaks. It became evident that the column was overloaded after a reduction in the number of beads analyzed produced better chromatographic peak shapes for these compounds. Use of only five beads and employment of a polar (Carbowax) column provides adequate peak shape, as can be seen in Figure 1. However, under the conditions of this work, where the Carbowax column was used for analysis and for cryofocusing, the stationary phase is not stable at the temperature of liquid nitrogen, nor at the high temperatures necessary to elute some compounds off of the column, such as the heavier fatty acid and lipid components. Due to these reasons, there is a significant background noticeable in the $\mathrm{PCl}$ and $\mathrm{NCI} \mathrm{RICs}$ of Figure 1. This background consists primarily of ions produced from column stationary-phase bleed. Comparison between the positive and negative ion $\mathrm{Cl}$ RICS in Figure 1 shows that the major peaks, which are carboxylic acids and lactic acid, can be correlated between these two ionization modes.

(21) Loeper, J. M . In M odern Practice of Gas Chromatography, 3rd ed.; Grob, R. L., Ed.; John Wiley \& Sons: New York, 1995; Chapter 14.

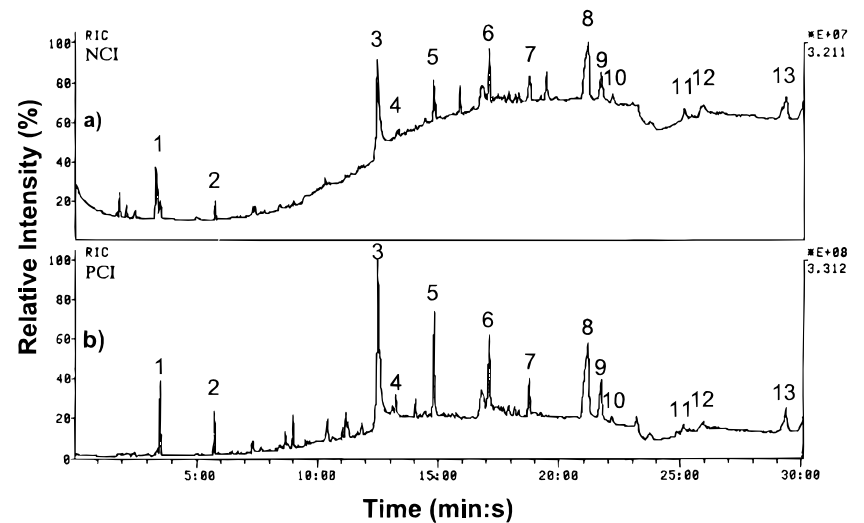

Figure 1. Reconstructed ion chromatograms of (a) $\mathrm{NCl}$ and (b) $\mathrm{PCl}$ from PPINCI analysis of thermally desorbed and cryofocused volatiles from five glass beads handled by a human subject. Separation by GC was effected by a $20 \mathrm{~m} \times 0.25 \mathrm{~mm}$ i.d. $\left(d_{\mathrm{f}}=0.25 \mu \mathrm{m}\right)$ Carbowax column. Compounds noted by peak numbers: (1) hexamethylcyclotrisiloxane, (2) octamethylcyclotrisiloxane, (3) lactic acid, (4) decanoic acid, (5) dodecanoic acid, (6) tetradecanoic acid, (7) pentadecanoic acid, (8) hexadecanoic acid, (9) hexadecenoic acid, (10) methyl hexadecanoate, (11) heptadecanoic acid, (12) heptadecenoic acid, and (13) octadecanoic acid.

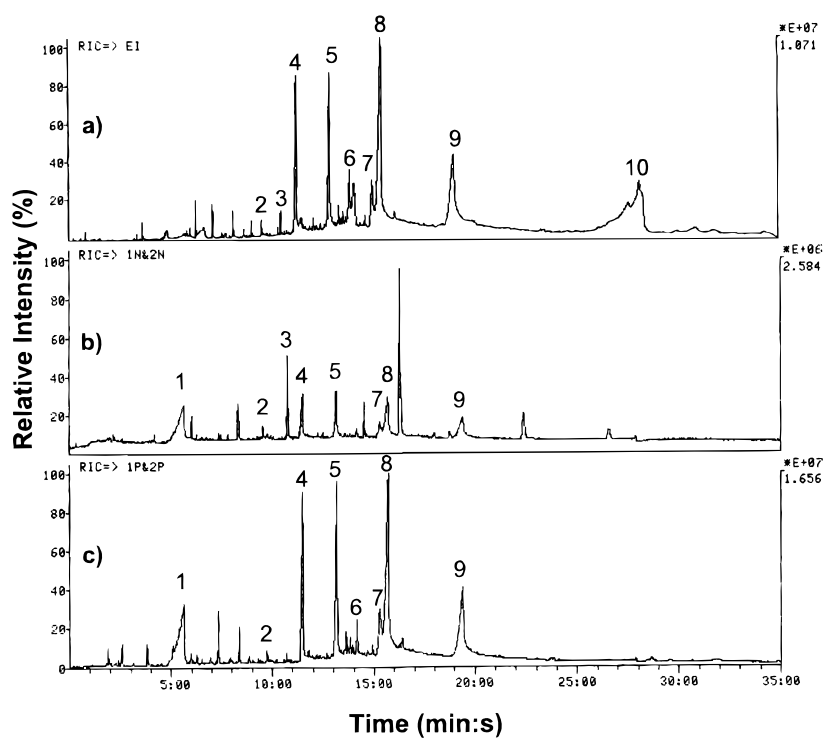

Figure 2. Reconstructed ion chromatograms of (a) $\mathrm{El}$, (b) $\mathrm{NCl}$ from $\mathrm{PPINICl}$, and (c) PCI from PPINICl analyses of thermally desorbed and cryofocused volatiles from five glass beads handled by the same human subject. Separation by GC was effected by a $25 \mathrm{~m} \times 0.20$ $\mathrm{mm}$ i.d. HP5 fused-silica open tubular column $\left(d_{\mathfrak{f}}=0.33 \mu \mathrm{m}\right)$. Compounds noted by peak numbers: (1) lactic acid, (2) decanoic acid, (3) decamethylcyclopentasiloxane, (4) dodecanoic acid, (5) tetradecanoic acid, (6) pentadecanoic acid, (7) hexadecenoic acid, (8) hexadecanoic acid, (9) octadecanoic acid, and (10) mono(2ethylhexyl)hexanedioic acid.

In later analyses, two columns of differing stationary phases were employed. Both HP5 and HP-FFAP columns were used in different experiments to effect the best separation of nonpolar and polar compounds, respectively. Chromatograms acquired with the HP5 column in EI and PPINICI modes are shown in Figure 2. The data contained in Figure $2 a$ were acquired from a later GC analysis, conducted on the same day and using conditions identical (other than ionization mode) to that of data in (b) and (c). When 


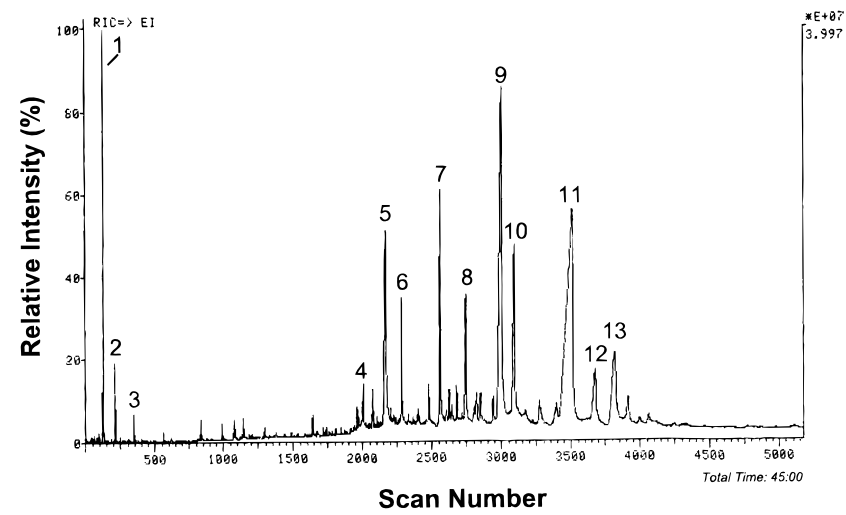

Figure 3. Reconstructed ion chromatogram of an El analysis of thermally desorbed and cryofocused volatiles from five glass beads handled by the same human subject, examined in the previous figures. Separation by GC was effected by a $25 \mathrm{~m} \times 0.20 \mathrm{~mm}$ i.d. HP-FFAP fused-silica open tubular column $\left(d_{\ddagger}=0.33 \mu \mathrm{m}\right)$. Compounds noted by peak numbers: (1) 1,1-difluoroethane, (2) hexamethylcyclotrisiloxane, (3) octamethylcyclotrisiloxane, (4) lactic acid, (5) glycerol, (6) dodecanoic acid, (7) tetradecanoic acid, (8) pentadecanoic acid, (9) hexadecanoic acid, (10) hexadecenoic acid, (11) octadecanoic acid, (12) octadecenoic acid, and (13) octadecadienoic acid.

the HP5 column was used, poor peak shape was evident for some polar components in the sample, e.g., lactic acid, observed as a fronted peak in Figure $2 b$ and $c$. However, it is easily identified in both $\mathrm{NCl}$ and $\mathrm{PCl}$ modes in the chromatograms by itscharacteristic fragmentation and adduct ions, which are discussed below.

Due to the number of polar compounds in these samples, a column with a more polar stationary phase (HP-FFAP) was used (Figure 3). In Figure 3, the peak shape of lactic acid is more symmetrical, although too small to see on this scale, than the peak of this compound observed with a HP5 column. Fronted peak shapes in the later eluting acids are due to overloading of the column, rather than due to the stationary phase. In addition to acids noted on the chromatogram, other compounds are also noted. There are siloxane stationary-phase peaks which appear early in the chromatogram. ${ }^{22}$ Additionally, residual 1,1-difluoroethane, which was used to cool the glass insert prior to handling, and a significant glycerol peak are present. It was later determined that the high amount of glycerol was due to the hair and scalp spray used by this volunteer.

Although the focus of this paper is on the method rather than the compounds identified, it is important to note that, for the most part, the chromatograms of emanations from different subjects are similar with respect to the compounds present (Figures 2 and 3). Human subjects differ mainly in the relative abundances of components and this has been an important factor from the biological standpoint of addressing mosquito host-location and the kairomones emanated by humans.

One of the major components found on human skin is L-lactic acid. Lactic acid is a known attractant of Ae. aegypti. ${ }^{4}$ This compound can be chromatographed by GC; however, lactic acid is extremely difficult to detect and identify by MS in EI mode (see Figures 2 and 3 ) due to the weak or absent molecular ion and the resultant fragment ions of low $\mathrm{m} / \mathrm{z}$. Fortunately, $\mathrm{NCl}$ can

(22) Barry, E. F. In M odern Practice of Gas Chromatography, 3rd ed.; Grob, R. L., Ed.; John Wiley \& Sons: New York, 1995; Chapter 3.

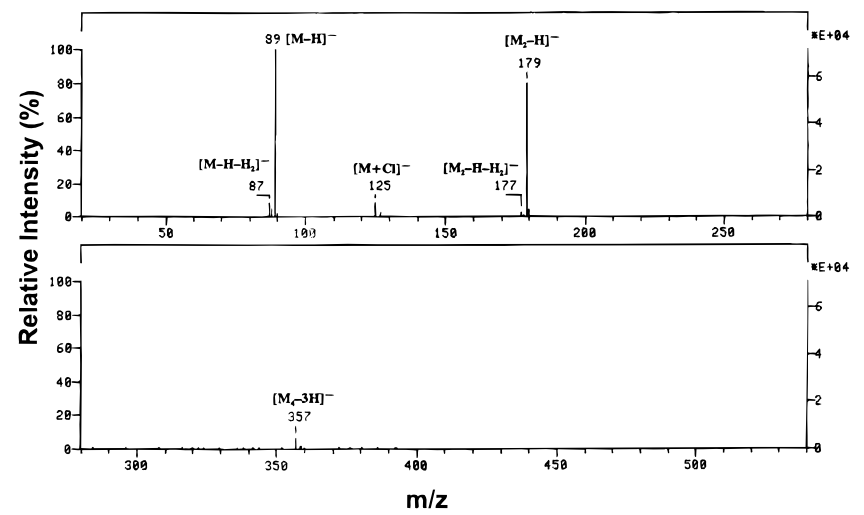

Figure 4. Negative ion mass spectrum of standard L-lactic Acid in methanol. A $0.5-\mu \mathrm{L}$ aliquot of a $120 \mathrm{ng} / \mu \mathrm{L}$ standard was injected to produce this mass spectrum.

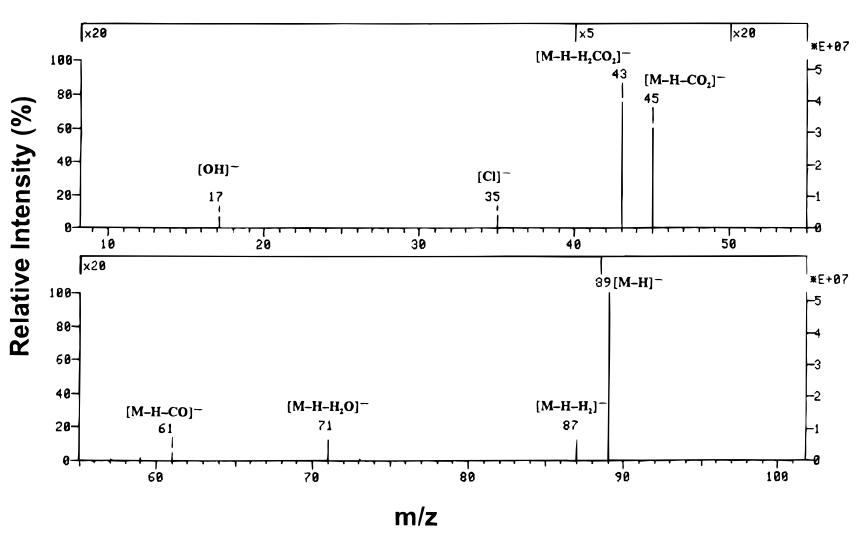

Figure 5. Negative ion product mass spectrum of the $[\mathrm{M}-\mathrm{H}]^{-}$ion of lactic acid observed from a sample of five glass beads handled by a human subject.

be used to identify, with little ambiguity, lactic acid in a sample. Analysis of the negative ions produced by lactic acid with a single stage of mass spectrometry can be used. This acid is susceptible to oligomerization in the ion source, readily undergoing self-Cl. This is not a desirable situation, but it is diagnostic, producing a $[\mathrm{M}-\mathrm{H}]^{-}$ion at $\mathrm{m} / \mathrm{z} 89, \mathrm{a}\left[\mathrm{M}_{2}-\mathrm{H}\right]^{-}$dimer ion at $\mathrm{m} / \mathrm{z} 179$, a sometimes absent, but weak if present $\left[\mathrm{M}_{3}-2 \mathrm{H}\right]^{-}$trimer ion at $\mathrm{m} / \mathrm{z} 268$, and $\mathrm{a}\left[\mathrm{M}_{4}-3 \mathrm{H}\right]^{-}$tetramer ion at $\mathrm{m} / \mathrm{z} 357$ (Figure 4). The trimer and tetramer ions undergo successive losses of water (18 Da), depending upon the amount of lactic acid present, the pressure, and the temperature in the ion source.

Tandem mass spectrometry can also be used to confirm the presence of lactic acid. In negative ion mode, the $[\mathrm{M}-\mathrm{H}]^{-}$ion at $\mathrm{m} / \mathrm{z} 89$ can be selected for CID. The product ion mass spectrum for this precursor ion of lactic acid is dominated by a loss of 44 $\left(\mathrm{CO}_{2}\right)$ and $46 \mathrm{Da}\left(\mathrm{H}_{2}+\mathrm{CO}_{2}\right)$, as seen in Figure 5. Alternatively, the protonated lactic acid, $[\mathrm{M}+\mathrm{H}]^{+}$ion at $\mathrm{m} / \mathrm{z} 91$ in $\mathrm{PCl}$ mode can be mass selected as the precursor ion and fragmented by CID. Examination for specific neutral losses from selected ions are also diagnostic in this case. Lactic acid is the only compound present in the volatiles from handled beads that has a $\mathrm{m} / \mathrm{z} 91$ precursor ion which fragments via a neutral loss of $28 \mathrm{Da}$ (CO) to form a product ion at $\mathrm{m} / \mathrm{z} 63$. Figure 6 a clearly shows that the neutral loss for this set of parameters does contain only one peak, that of lactic acid. Other compounds in the sample that have a carbonyl moiety, such as carboxylic acids, may undergo CID via 


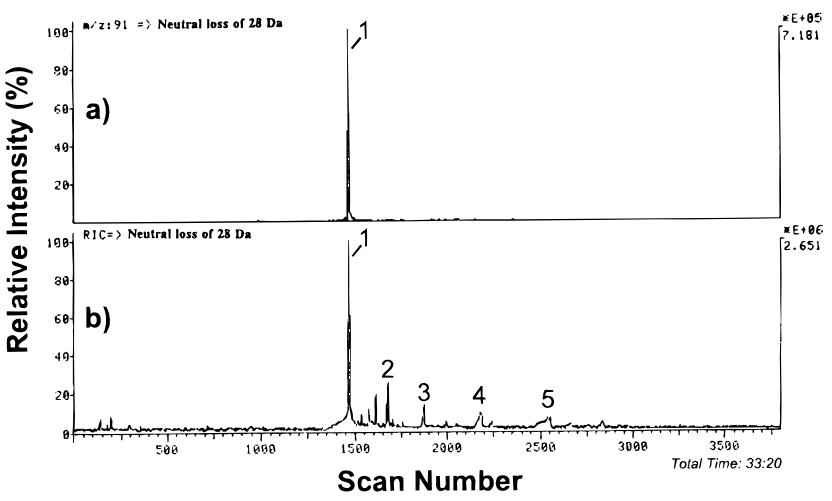

Figure 6. (a) Positive ion mode neutral loss reconstructed ion chromatograms of a neutral loss of $28 \mathrm{Da}$ from an ion with $\mathrm{m} / \mathrm{z} 91$. This ion corresponds to protonated lactic acid, formed in the ion source. (b) Positive ion mode neutral loss reconstructed ion chromatograms of $28 \mathrm{Da}$ from precursor ions of $\mathrm{m} / \mathrm{z} 35-650$ for the duration of sample analysis. Peaks shown have one or more ions that produce, upon CID, a product ion that is $28 \mathrm{Da}$ less than the precursor ion. These were obtained using a $25 \mathrm{~m} \times 0.20 \mathrm{~mm}$ i.d. $\left(d_{\mathrm{f}}\right.$ $=0.33 \mu \mathrm{m}) \mathrm{HP}-\mathrm{FFAP}$ column. The sample consisted of five beads handled by a human subject. The displayed range encompasses the first 33:20 min of a 40:00-min analysis. Compounds noted by peak numbers: (1) lactic acid, (2) dodecanoic acid, (3) tetradecanoic acid, (4) hexadecanoic acid, and (5) octadecanoic acid.

a neutral loss of $28 \mathrm{Da}$, as well (Figure 6b). However, the peaks attributable to carboxylic acids in the sample can be made readily apparent by also scanning for neutral losses of 46, 30, and $18 \mathrm{Da}$ in $\mathrm{PCl}$ mode, as shown in parts $\mathrm{a}-\mathrm{c}$ of Figure 7 , respectively. The neutral loss of $46 \mathrm{Da}\left(\mathrm{H}_{2}+\mathrm{CO}_{2}\right)$ is indicative of carboxylic acids alone, and the neutral losses of $30\left(\mathrm{H}_{2}+\mathrm{CO}\right)$ and $18 \mathrm{Da}$ $\left(\mathrm{H}_{2} \mathrm{O}\right)$ are presented as additional confirmation of the presence of this compound class. It should be noted that other classes, such as aldehydes and ketones, undergo neutral losses of $30 \mathrm{Da}$ and that ketones and alcohols also undergo neutral losses of $18 \mathrm{Da}$.

Microscale Purge and Trap GC Analysis. M icroscale purge and trap was initially avoided in this study due to irreversible adsorption of highly polar compounds in one of the traps. However, upon analysis via a three-stage microscale purge and trap GC/MS system, it was found that a number of trace components could be concentrated and identified, as readily seen in the $\mathrm{EI}$ reconstructed ion chromatogram of Figure 8. Data for this figure were acquired by thermal desorption of volatiles from 100 handled beads held in a heated round-bottom flask. The entire chromatogram consists of components that elute prior to tetradecane on a DB-1 column. Based on the thermal desorption/ cryofocusing $\mathrm{GC}$ results (Figures $1-3$ ), the aliphatic fatty acids are the most abundant components in emanations transferred to glass from the skin. Their removal in the purge and trap system permits the analysis of trace level components constituting other compound classes, such as the aldehydes, which are found in greatest abundance for this method, as seen in this figure. Some of the trace level components may assist the mosquitoes in the host location process. M ore detailed analysis of these components will be presented in a subsequent publication.

\section{CONCLUSIONS}

The cyrofocused GC/MS chromatograms of human skin emanations contain over 300 observable compounds; these will

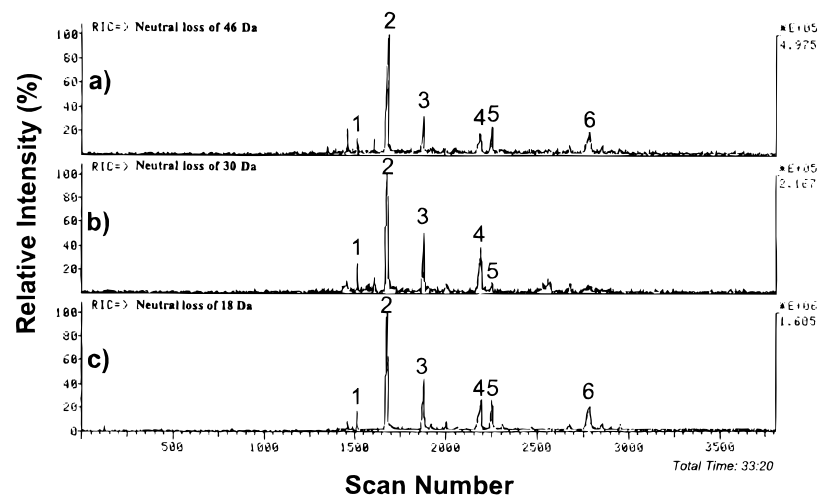

Figure 7. Positive ion mode neutral loss reconstructed ion chromatograms of (a) $46 \mathrm{Da}$, indicative of carboxylic acids, (b) $30 \mathrm{Da}$, indicative of carboxylic acids, aldehydes, and ketones, and (c) 18 $\mathrm{Da}$, indicative of carboxylic acids, alcohols, and ketones. Separation was effected by a $25 \mathrm{~m} \times 0.20 \mathrm{~mm}$ i.d. $\left(d_{f}=0.33 \mu \mathrm{m}\right) \mathrm{HP}-\mathrm{FFAP}$ column. The sample consisted of five beads handled by a human subject. The displayed range encompasses the first 33:20 min of a 40:00-min analysis. Compounds noted by peak numbers: (1) lactic acid, (2) dodecanoic acid, (3) tetradecanoic acid, (4) hexadecanoic acid, (5) hexadecenoic acid, and (6) octadecanoic acid.

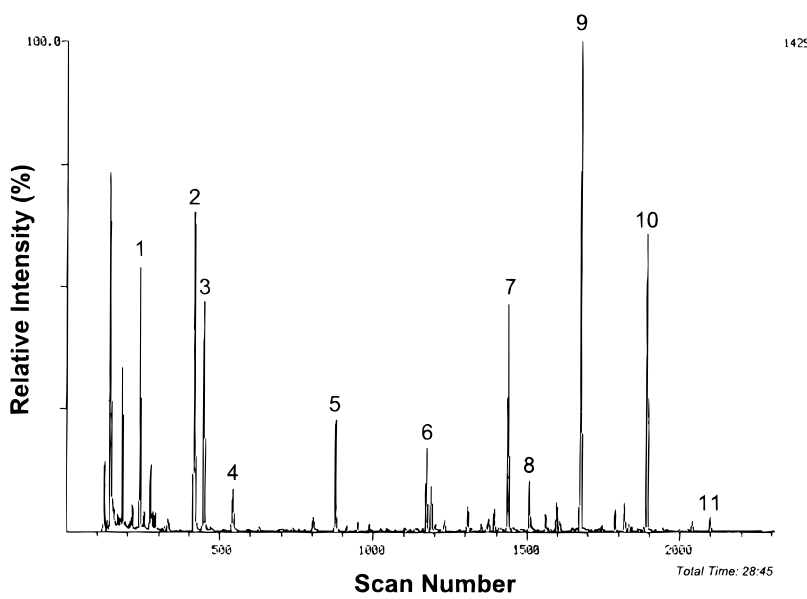

Figure 8. Reconstructed ion chromatogram from $\mathrm{El}$ analysis of volatiles desorbed from 100 handled glass beads. A three-stage purge and trap system, employing a $30 \mathrm{~m} \times 0.25 \mathrm{~mm}$ i.d. $\left(d_{\mathrm{f}}=1.0 \mu \mathrm{m}\right)$ DB-1 column, was used for the analysis. Compounds noted by peak numbers: (1) butanal, (2) 3-methylbutanal, (3) 2-methylbutanal, (4) pentanal, (5) hexanal, (6) heptanal, (7) octanal, (8) phenylacetaldehyde, (9) nonanal, (10) decanal, and (11) undecanal.

be reported in a forthcoming paper. The GC/ M S profiles are well resolved in both the cryofocused and the microscale purge and trap experiments; however, many of the compounds detected differ qualitatively and quantitatively between these methods. The sampling methods satisfy the initial criterion of detecting or identifying volatile emanations in a manner similar to that which mosquitoes encounter. Specifically, this refers to sampling a chemically unmodified sample volatilized into the gas phase. Purge and trap introduction was employed as an alternative, complementary method to detect volatile components; furthermore, it discriminates against polar compounds such as fatty acids, which are predominant in cryofocused analyses. This allows for detection of some trace components (highly volatile nonpolar to moderately polar) not readily detected in the cryofocused analyses.

The use of glass beads to collect animal emanations, or any host emanations for other kairomone studies, is also possible, 
provided that the active components are transferrable to glass. The primary benefit of this method is the ability to perform a solventless load of volatiles onto a GC column, with minimal effort and expense, and without excessive deposition of water.

\section{ACKNOWLEDGMENT}

Funding for this work was supplied by a specific cooperative research grant (58-6615-6-021) from the Mosquito and Fly Re- search Unit at the USDA-ARS-CM AVE. The authors thank Carl Schreck, Daniel Kline, and Donald Barnard of the USDA for their assistance.

Received for review September 3, 1998. Accepted November 5, 1998.

AC980990V 